\title{
Controlling intermediate dynamics in a family of quadratic maps
}

\author{
Rafael M. da Silva ${ }^{1}$, Cesar Manchein ${ }^{2}$, and Marcus W. Beims ${ }^{1}$ \\ ${ }^{1}$ Departamento de Física, Universidade Federal do Paraná, 81531-980 Curitiba, PR, Brazil and \\ ${ }^{2}$ Departamento de Física, Universidade do Estado de Santa Catarina, 89219-710 Joinville, SC, Brazil
}

(Dated: October 17, 2018)

\begin{abstract}
The intermediate dynamics of composed one-dimensional maps is used to multiply attractors in phase space and create multiple independent bifurcation diagrams which can split apart. Results are shown for the composition of $k$-paradigmatic quadratic maps with distinct values of parameters generating $k$-independent bifurcation diagrams with corresponding $k$ orbital points. For specific conditions, the basic mechanism for creating the shifted diagrams is the prohibition of period doubling bifurcations transformed in saddle-node bifurcations.
\end{abstract}

PACS numbers: 05.45.Ac, 05.45.Pq

Keywords: Quadratic map, chaos, bifurcation.

In generic dynamical systems it is very useful to determine the right parameter combination for which regular or chaotic behavior takes place. It is more interesting to know or control the robustness of the dynamics under certain time parametric changes. With this aim in mind we present in this work a recipe to perform time parametric changes in order to control the intermediate dynamics of composed maps. By using the composition of a family of quadratic maps, it is possible to generate multiple shifted similar independent bifurcation diagrams and by consequence the same number of attractors in phase space. We show that for specific parameter combinations occurs a prohibition of period doubling bifurcations and the appearance of extra-stable motion. As unimodal maps follow universal bifurcation rules we believe that the proposed method is generic and may be extended to ordinary higher-dimensional problems involving nonlinear behaviours.

\section{INTRODUCTION}

Nonlinear dynamical systems are one of the most important tools to model a large number of physical systems in nature, ranging from biological populations, coupled networks, market crisis, brain dynamics, chemical systems, laser physics, granular dynamics, normal and anomalous transport, extreme events, weather forecast, among many others. The parameter values in such physical systems deliberate the underlying complex dynamics. One of the greatest challenge in nonlinear models is to identify what are the correct parameter values which leaves to the desired dynamics (or avoid it) and if these values follow some kind of universal rule.

Once the above mentioned desired parameter combination is found, we ask what is the robustness of the dynamics under time parametric changes. This is of relevance for practical purposes where nonlinear models describe realistic problems. In this context, weak parametric perturbations were used to control the chaotic motion [1-3], to mention a few. In the present work we use the concept of parametric changes to control the intermediate dynamics (defined in Sec. II) of composed maps. Our goal is to analyse the whole change in the dynamics, including regular and chaotic motions. We show that using the composition of a family of quadratic maps [4], it is possible to generate multiple shifted independent bifurcation diagrams and the same number of attractors in phase space. For specific cases discussed later, we show that the mechanism of shifted bifurcation diagrams and prohibition of period doubling bifurcations are responsible for the appearance of extra-stable motion. An analogous mechanism for just two shifted bifurcation diagrams was revealed many years ago [3] in the context of taming chaos in continuous-time systems under weak harmonic perturbation (see also Ref. [5]). In the context of chaos suppression, a similar method was used in the particular case of duplication in the factorization of two quadratic coupled maps [6] and one-dimensional maps were also used for applications of feedback and non-feedback control techniques [7-9]. Periodic perturbations can also be applied to determine the role of extreme orbits in the organization of periodic regions in the parameter space of one dimensional maps [10].

Our results are not restricted to weak parametric perturbations and we show cases of duplication, triplication, quadruplication and quintuplication of bifurcation diagrams and corresponding attractors. In addition, since we use the composition of unimodal maps, which have only one critical point and display very similar dynamical behaviour, the proposed method is generic and can be applied to ordinary higher-dimensional problems involving nonlinear behaviours.

The remainder of this paper is organized as follows. In Sec. II the general concept of intermediate dynamics is given in terms of the composition of maps. Section III is devoted to discuss the main mechanism which induces the shift of bifurcations diagrams in the quadratic map, and consequently the proliferation of periodic attractors in its phase space. In addition, the application to du, tri, quadru and quintuplicate periodic attractors is discussed in the same section. Finally, in Sec. IV we summarize 
our results indicating some of their possible implications.

\section{INTERMEDIATE DYNAMICS}

The key idea to generate multiple attractors is to control the dynamics of intermediate variables of composed maps. To explain this in details consider a onedimensional discrete map given by

$$
x_{n+1}=f\left(x_{n}, \alpha\right) \text {, }
$$

with $n=0,1,2, \ldots$ being the discrete times, $x_{n}$ the state of the system at time $n$ whose time evolution is described by the function $f$ (usually nonlinear) and $\alpha$ representing all involved parameter. Now, we construct a composed map obtained by applying the map (1) $k$-times using distinct parameters at each iteration, namely

$$
x_{n+k}^{(c)}=f\left(x_{n+k-1}, \alpha_{k}\right) \circ \ldots \circ f\left(x_{n+1}, \alpha_{2}\right) \circ f\left(x_{n}^{(c)}, \alpha_{1}\right),
$$

where parameters $\alpha_{1}, \alpha_{2}, \ldots \alpha_{k}$ follow a specific protocol defined later. In this work the superscript (c) indicates quantities related to the composed map. All

We start with the most simple case of duplication, where the time parameter variation has a period $k=2$. In such case the modified quadratic map (MQM) is defined as

$$
x_{n+1}=a-x_{n}^{2}+F(-1)^{n},
$$

with $F$ being the intensity which changes the dynamics for each iteration and the signal $(+)$ is used for $n$ even and $(-)$ for $n$ odd. The composed map

$$
x_{n+2}^{(c)}=a-\left[a-\left(x_{n}^{(c)}\right)^{2}+F\right]^{2}-F,
$$

is a composition of two MQMs with alternating values of $F$, namely $+F,-F,+F,-F, \ldots$. In fact, in this case the intermediate dynamics corresponds to a quadratic map $x_{n+1}^{\prime}=a_{n}^{\prime}-x_{n}^{\prime 2}$ with time dependent parameter $a_{n}^{\prime}=a+F(-1)^{n}$. A per- $1^{c}$ orbit from the composed map obeys $x_{2}^{(c)}=a-x_{1}^{2}-F=x_{0}^{(c)}$ with $x_{1}=a-\left[x_{0}^{(c)}\right]^{2}+F$ and $x_{2}^{(c)}$ is the fixed point from the composed map. The intermediate point $x_{1}$ is not a fixed point from the composed map but it is necessary to realize the connection $x_{0}^{(c)} \rightarrow x_{1} \rightarrow x_{2}^{(c)}=x_{0}^{(c)}$.

It is interesting to observe the bifurcation diagram for the composed map (4) with $F=0$, plotted in Figs. 1(a)(c) (black and green curves). The shape of this bifurcation diagram is identical to the bifurcation diagram for the usual QM, Eq. (3) with $F=0$, with one essential difference explained next. It is known that for the states from $x_{n+1}$ to $x_{n+k-1}$ are called intermediate states. The dynamics of each intermediate state can be controlled and manipulated independently. As we will see, $k$-independent intermediate dynamics (chaotic or regular) are generated. It could also be possible to use distinct functions $f$ at each intermediate dynamics, but such case will not be discussed here. Another approach would be to define new variables for each intermediate dynamics, which corresponds to consider a higher-dimensional complex system.

The concept of intermediate dynamics has been used in open chaotic flows to describe the particle's position after a half period [11] and in chemical oscillations where the concentrations of intermediates reactants were allowed to vary [12]. Not unintentional, the composition used in this work is analogous to external controlling forces and can promptly be implemented in experiments. Some of the related references are given in Sec. I.

\section{COMPOSED QUADRATIC MAPS}

$$
\text { A. Duplication }(k=2)
$$

usual QM a period-1 (shortly written as per-1) is born at $a_{1}=-0.25$, and period doubling bifurcations (PDBs) $1 \rightarrow 2$ and $2 \rightarrow 4$ occur respectively at $a_{12}=0.75$ and $a_{24}=1.25$. For the composed map, inside the interval $\left[a_{12}, a_{24}\right]$, instead a per- 2 orbit, we have two per- $1^{c}$ orbits, one drawn in black and the other one in green. Thus, to obtain the bifurcation diagram for the composed map it is necessary to use more ICs.

Now we use $F=7 \times 10^{-3}$ to analyze the bifurcation diagram for the composed map in comparison with the QM. This is shown in Fig. 1(a), blue curve for the IC used to obtain the black curve from the $F=0$ case, and red curve for the IC related to the green curve from the $F=0$ case. Thus the per- 1 from the QM is transformed in a per- $1_{1}^{c}$ (blue curve) orbit from the composed map. This orbit does not suffer a PDB at $a_{12}=0.75$ anymore, but at $a_{12} \sim 1.23$. The PDB at $a_{12}=0.75$ becomes forbidden for the composed map and the per$1_{1}^{c}$ avoids the bifurcation point at $a_{12}$. Instead, a new per $1_{2}^{c}$ (red curve) orbit is born at $a=0.820$ (see the black-continuous line in Figs. 1(a)-(c)). Actually, it is a structural change in the dynamics when compared to the $F=0$ case. The per- $1_{1}^{c}$ and per- $1_{2}^{c}$ orbits are independent (different ICs), have distinct stabilities and suffer a PDB $1^{c} \rightarrow 2^{c}$ at distinct values of $a$, namely per- $1_{1}^{c}$ at $a=1.235$ and per- $1_{2}^{c}$ at $a=1.265$, as represented in Fig. 1 by the black dotted and black dashed lines, respectively. This is the explanation for the shift (to smaller and larger values of $a$ ) in the bifurcation di- 

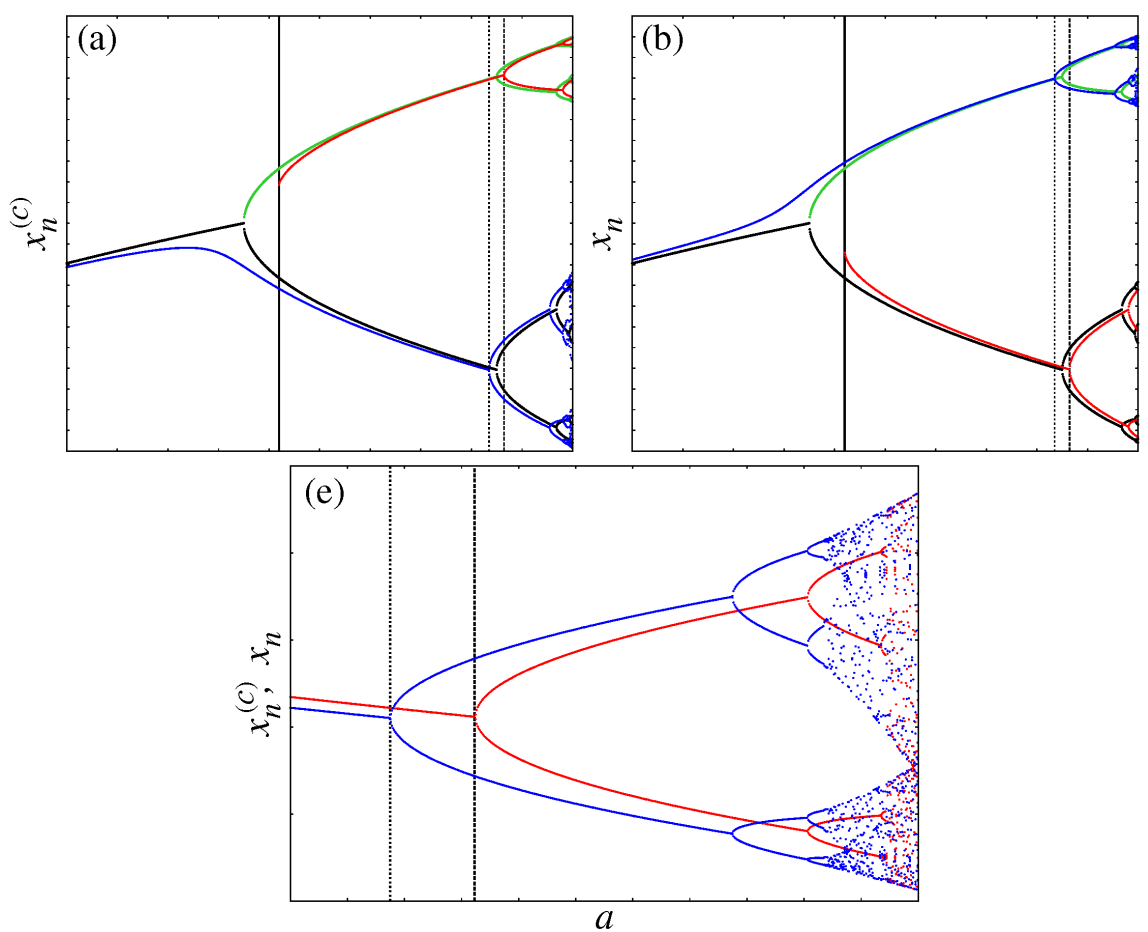
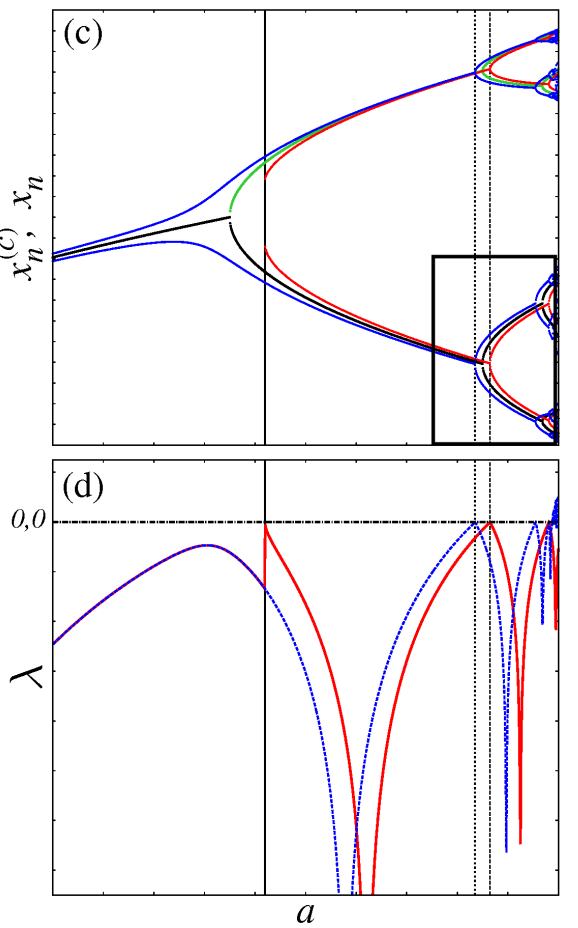

Figure 1. (Color online) Bifurcation diagram for the composition of MQMs with $k=2$. The black and green curves represent different attractors for the case $F=0$ while red and blue curves represent different attractors for the case $F=7 \times 10^{-3}$. In (a) the composed map $x_{n}^{(c)}$ (see text) is plotted, in (b) only the intermediate points $x_{n}$ are plotted and in (c) $x_{n}^{(c)}$ and $x_{n}$ are displayed together, all these cases for the interval $\left(a_{\min }, a_{\max }\right)=(0.4,1.4)$ and $\left(x_{\min }, x_{\max }\right)=(-0.6,1.5)$. In $(\mathrm{d})$, the Lyapunov exponent for the composed map $x_{n}^{(c)}$ is shown for same interval of $a$ and $\left(\lambda_{\min }, \lambda_{\max }\right)=(-3.0,0.5)$, demonstrating the occurrence of bifurcations $(\lambda=0)$ for the values of $a$ indicated by the vertical lines. In (e) we show the magnification of the black box in (c) to evidence the shifted and duplicated bifurcation diagram when $x_{n}^{(c)}$ and $x_{n}$ are considered for $F \neq 0$.

agram and it is essentially related to the prohibition of a PDB at $a_{12}$ due to $F \neq 0$. Figure 1(b) shows the bifurcation diagram for the intermediate dynamics $x_{n}$. Essentially it has a similar (mirror symmetric) behavior than those shown in Fig. 1(a). Thus we have generated, including the intermediate points, two independent and shifted bifurcation diagrams as shown in Fig. 1(c) and magnified in Fig. 1(e). The Lyapunov exponent from all orbital points for the composed map is shown in Fig. 1(d). It is worth to mention that if we use the sequence $-F,+F,-F,+F, \ldots$ instead $+F,-F,+F,-F \ldots$, intermediate and orbital points are switched, keeping exactly the same properties discussed above.

At this stage, a natural question that arises is related to how the composition of one-dimensional maps perturbs the position of fixed points in phase and parameter spaces. In order to clarify this issue, we derive analytical expressions for the orbits of the composed map for the shifted bifurcation parameter. For the born of per- $1_{1}^{c}$ and per- $1_{2}^{c}$ orbits via saddle-node bifurcation the expression is

$$
\begin{aligned}
W_{1^{c}}^{(k=2)}(a, F)= & -256 F^{4}+\left(512 a^{2}+1536 a+288\right) F^{2} \\
& -W_{1}(a) W_{1 \rightarrow 2}(a)=0
\end{aligned}
$$

while for the PDB $1^{c} \rightarrow 2^{c}$ it is given by

$$
\begin{aligned}
W_{1^{c} \rightarrow 2^{c}}^{(k=2)}(a, F)= & -256 F^{4}+\left(512 a^{2}+1536 a+160\right) F^{2} \\
& -W_{2 \rightarrow 4}(a)=0 .
\end{aligned}
$$

Equations (5) and (6) are written as functions of the boundary conditions in parameter space from the usual QM, namely $W_{1}(a)=(4 a+1)=0 \rightarrow(a=-1 / 4)$ which gives the saddle-node bifurcation, $W_{1 \rightarrow 2}(a)=(4 a-3)^{3}=$ $0 \rightarrow(a=3 / 4)$ the $\mathrm{PDB} 1 \rightarrow 2$ and $W_{2 \rightarrow 4}(a)=$ $\left(16 a^{2}+8 a+5\right)(4 a-5)^{2}=0 \rightarrow(a=5 / 4,-1 / 4 \pm i / 2)$ the PDB $2 \rightarrow 4$ (see [13] for details how to obtain such boundary conditions in parameter space). Note that the composed map couples $W_{1}(a)$ and $W_{1 \rightarrow 2}(a)$ and the PDB $1 \rightarrow 2$ is now inserted into the expression (5) which is for the saddle-node bifurcation of per- $1_{1}^{c}$ and per- $1_{2}^{c}$ orbits. Thus, the PDB from the QM is transformed into a saddlenode bifurcation for the composed map, which demonstrates analytically the prohibition of the PDB $1 \rightarrow 2$. For the case discussed above, with $F=7 \times 10^{-3}$, the solutions of $W_{1^{c}}^{(k=2)}$ and $W_{1^{c} \rightarrow 2^{c}}^{(k=2)}$ are, respectively, $a=-0.249$ (born of per- $1_{1}^{c}$, blue curve), $a=0.820$ (born of per$1_{2}^{c}$, red curve) and $a_{12}=1.235,1.265$, where the PDBs $1^{c} \rightarrow 2^{c}$ of per- $1_{1}^{c}$ and per- $1_{2}^{c}$, respectively, occur. Note that the complex solution $-1 / 4 \pm i / 2$ from $W_{2 \rightarrow 4}(a)=0$ 


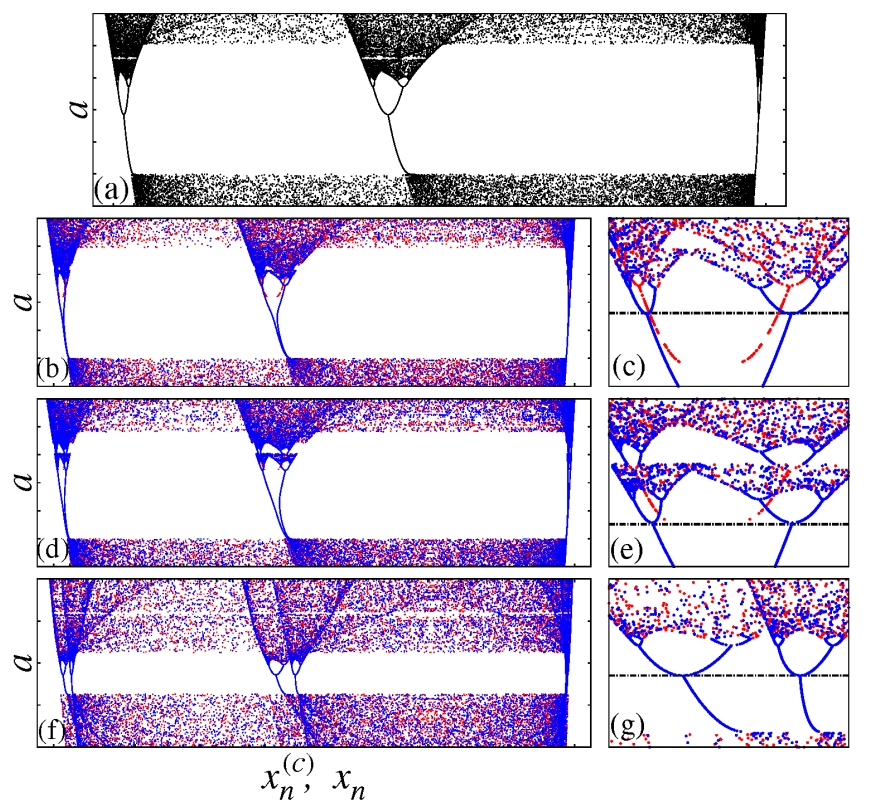

Figure 2. (Color online) Bifurcation diagram for the composition of MQMs with $k=2$ (sequence $+F,-F,+F,-F, \ldots$ ) plotting the composed map $x_{n}^{(c)}$ and intermediate points $x_{n}$, showing a per-3 window for (a) $F=0$, (b) and (c) $F=$ $3 \times 10^{-4}$, (d) and (e) $F=7 \times 10^{-4}$, (f) and (g) $F=8 \times 10^{-3}$. The black horizontal lines in (c), (e) and (g) indicate the PDB $1 \rightarrow 2$ that occurs at the same value of $a$ for each branch of the per-3 window of the $F=0$ case. In (a), (b), (d) and (f) the interval of $x$ and $a$ used are $\left(x_{\min }, x_{\max }\right)=(-1.50,1.90)$ and $\left(a_{\min }, a_{\max }\right)=(1.74,1.80)$. is transformed into a real solution leading to two PDBs $1^{c} \rightarrow 2^{c}$. This proves analytically the origin of shifted bifurcation diagrams at distinct values of $a_{12}$.

Now we present details for the case $k=2$ applied to a per-3 window of the QM in Fig. 2. Figs. 2(b), (d) and (f) show the bifurcation diagram for increasing values of $F$ and Figs. 2(c),(e) and (g) display corresponding magnifications. As expected, the per-3 orbit is transformed in a per- 6 one, i.e., a per- $3^{c}$ orbit of the composed map, and the usual PDB $3 \rightarrow 6$ of the QM becomes prohibited (see Fig. 2(c)), as in the per-1 case discussed above. In this case, the duplication process is only allowed in the region of per- 6 orbit of the QM, after the PDBs indicated by the horizontal lines in Figs. 2(c), (e) and (g). Therefore, we have three additional shifted and partially superposed bifurcation diagrams which for the largest values of $F$ in Fig. 2(f), are embedded in the chaotic zone. This is the reason of stabilization and suppression of the chaotic motion, i.e., periodic and chaotic attractor are coexisting in the same set of parameter $a$. With these results we conclude that the shift in the bifurcation diagram and the duplication of stable regions is only allowed for even periods when $k=2$, while odd periods $p$ becomes $p^{\prime}=2 p$ (per-1 becomes per-2 in Fig. 1 and per- 3 becomes per- 6 in Fig. 2), but are not shifted.

B. Triplication $(k=3)$
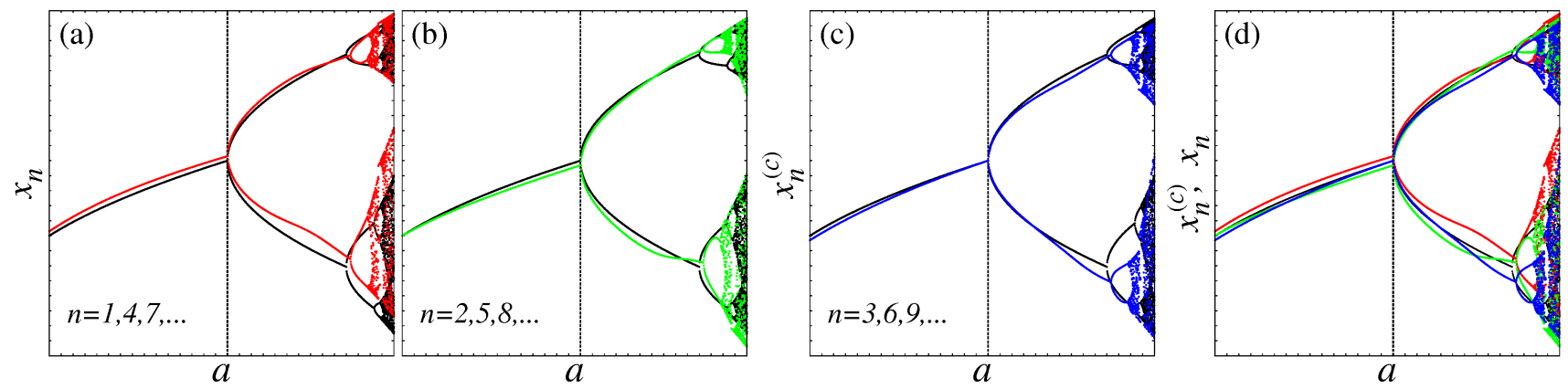

Figure 3. (Color online) Bifurcation diagrams for a sequence $+F, 0,-F(k=3)$ with $F=3 \times 10^{-2}$ (colored dots) using the intermediate points $x_{n}$ in (a)-(b), the composed map $x_{n}^{(c)}$ in (c) and both in (d). The black dots represent the bifurcation diagram for the QM in all panels. The parameter $a$ and variable $x$ range used in all bifurcation diagrams $\operatorname{are}\left(a_{\min }, a_{\max }\right)=(0.00,1.45)$ and $\left(x_{\min }, x_{\max }\right)=(-0.80,1.50)$, respectively.

To understand more complex behaviours, it is necessary to discuss in details the case of triplication. With this purpose in mind we perturb the dynamics of the QM by using the $k=3$ sequence $-F, 0,+F,-F, 0,+F \ldots$

The per-1 orbit from the composed map becomes a composition of three orbital points from the intermediate dynamics. The intermediate dynamics can be observed in Figs. 3(a)-(b) while the composed map is plotted in Fig $3(\mathrm{c})$ and the total dynamics is displayed in Fig. 3(d). In Figs (a)-(c) only the iterations $n=1,4,7, \ldots($ for $+F)$, 

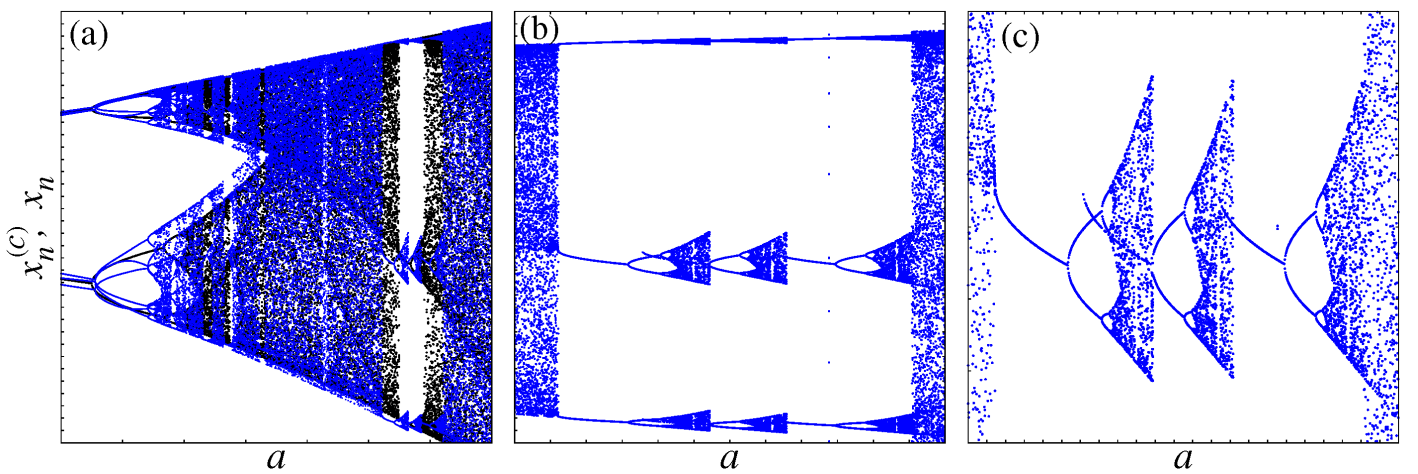

Figure 4. (Color online) Bifurcation diagram for the composition of MQMs using a sequence $+F, 0,-F(k=3)$ which generates the triplication of a per-3 window of the QM. In (a) the QM (black dots), the composed map $x_{n}^{(c)}$ and intermediate points $x_{n}$ (blue dots) for $F=1.5 \times 10^{-3}$ are plotted in the interval of $\left(a_{\min }, a_{\max }\right)=(1.2,1.9)$ and $\left(x_{\min }, x_{\max }\right)=(-1.5,2.0)$, while in (b) only $x_{n}^{c}$ and $x_{n}$ are plotted showing the triplication of the per-3 window (or per- 1 of the composed map) in the interval of $\left(a_{\min }, a_{\max }\right)=(1.71,1.83)$ and $\left(x_{\min }, x_{\max }\right)=(-1.5,2.0)$. Panel $(\mathrm{c})$ is a magnification of small portion of Fig. (b) for the interval $\left(a_{\min }, a_{\max }\right)=(1.715,1.830)$ and $\left(x_{\min }, x_{\max }\right)=(-0.3,0.3)$ where three similar small bifurcation diagrams are generated by the MQM.

$n=2,5,8, \ldots($ for 0$)$ and, $n=3,6,9, \ldots($ for $-F)$ are plotted in red, green and blue dots, respectively. For comparison, all bifurcation diagrams are superposed to the bifurcation diagram of the QM, plotted with black dots. In this scenario the PDB is not prohibited and

However, shifted bifurcations may occur for other periodic orbits, namely, periods multiple of 3 . To clarify this process a larger portion of the bifurcation diagram is magnified in Fig. 4(a), which includes a per-3 window from the QM in black and the composed map in blue dots. For smaller values of $a$ there are no shifted bifurcation diagrams, as previously shown in Fig. 3(d). On the other hand, as $a$ increases, some regular windows are already shifted when compared to the $F=0$ case. The reason for these shifts are explained using the larger per3 window, which are magnified in Figs. 4(b) and nicely visualized in (c). As observed for the duplication of per-2 attractors, in the triplication of per-3 orbital points, three shifted saddle-node bifurcations occur, while for the QM only one saddle-node bifurcation gives rise to the per3 window for $a=7 / 4$. It is important to note that the per-3 window is enlarge and the period remains the same, while for other periods $p$, that are not multiple of $k=3$, become $p^{\prime}=3 p$ when $F \neq 0$.

\section{Quadruplication and quintuplication}

In the present Section the recipe discussed above is extended to quadruplicate and quintuplicate attractors in phase space to obtain similar shifted bifurcation diagrams. For the quadruplication the bifurcation points are no shifted as before. Only the phase space is composed of more stable points (or periodic attractors). The stable regimes in the parameter $a$ remain unaltered.

and quintuplication processes we use the protocols $+F,-F / 2,+F / 2,-F,+F,-F / 2, \ldots(k=4)$ and $+F,-F / 2,0,+F / 2,-F,+F,-F / 2, \ldots(k=5)$, respectively. For $k=4$, the multiplication of stable regions occurs as in the case $k=2$. However not only the PDB $1 \rightarrow 2$ is prohibited but also $2 \rightarrow 4$, giving rise to four saddle-node bifurcations, displayed by vertical dotted lines in Figs. 5(a) and (b). The case $k=5$ can be compared to the case $k=3$ and it is shown in Fig 5(c) $(F=0)$ and $(\mathrm{d})(F \neq 0)$.

\section{CONCLUSIONS}

In this work we show that the control of intermediate dynamics can be used to enlarge stable domains in phase and parameter spaces of nonlinear dynamical systems. We present analytical and numerical results for the specific case of the composition of quadratic maps with distinct parameters. In general, performing many simulations we observed that the sign of the perturbation must change for each composition of the map. In addition, the $k$-compositions generate $k$ attractors and $k$-shifted independent bifurcations diagrams when $\omega \in \mathbb{Z}$, where $\omega=p / k$ and $p$ being the period of the orbits from the QM. For $F \neq 0$ the original period of the orbit remains equal. When $\omega \notin \mathbb{Z}$ we still generate $k$-attractors, 

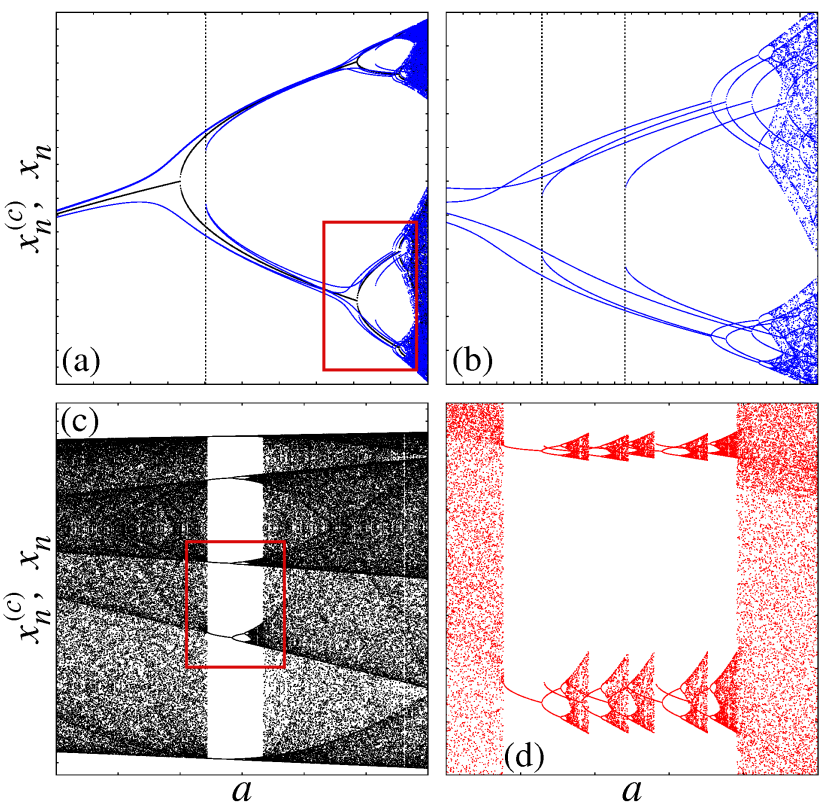

Figure 5. (Color online) Bifurcation diagram for the quadruplication and quintuplication of the QM plotting $x_{n}^{(c)}$ and $x_{n}$. In (a) the quadruplication is shown for $F=1 \times 10^{-2}$ (blue curves) inside the interval $\left(a_{\min }, a_{\max }\right)=(0.40,1.45)$, $\left(x_{\min }, x_{\max }\right)=(-0.70,1.50)$. For comparison the bifurcation diagram for the QM is shown with a black curve. In (b) the magnification of the region inside the red box of (a) is plotted and black dotted lines indicate the saddle-node bifurcations that create new attractors. In (c) a per-5 window of the QM is plotted inside the interval $\left(a_{\min }, a_{\max }\right)=(1.60,1.66)$, $\left(x_{\min }, x_{\max }\right)=(-1.15,1.90)$. In (d) the quintuplication of two orbital points [see red box in (c)] and the corresponding shifted bifurcation diagrams are shown for $F=4 \times 10^{-3}$. but the bifurcation diagrams are not shifted anymore. In this case the period of the orbits obeys $p^{\prime}=p k$ since it counts also the intermediate orbits.

The ability to control the intermediate dynamics in realistic situations has relevant implications: (i) multiple composition of maps lead to the appearance of multiple shifted bifurcation diagrams. Consequently occurs a considerable enlargement of the stable domains in phase and parameter spaces. This is crucial for the survival of the desired dynamics under noise and temperature effects, which usually destroy periodic motion [14, 15]; (ii) the number $k$ of modified maps used in the composition determinates the period- $p$ of multiplied bifurcation diagrams since the necessary condition is that $\omega \in \mathbb{Z}$, with $\omega=p / k$.

The easy way of generating and moving shifted bifurcation diagrams by controlling the intermediate dynamics of composed maps is definitely the remarkable contribution of the present work. Consequences of shifted bifurcations in two dimensional systems were analyzed recently by multiplying isoperiodic stable structures in the parameter space of the Hénon map [16]. Future contributions intend to verify to which extend such multiplication of stable motion can be realized in the parameter space of classical [17, 18] and quantum ratchets [19].

\section{ACKNOWLEDGMENTS}

R.M.S. thanks CAPES (Brazil) and C.M. and M.W.B. thank $\mathrm{CNPq}$ (Brazil) for financial support. C.M. also thanks FAPESC (Brazil) for financial support. The authors also acknowledge computational support from Professor Carlos M. de Carvalho at LFTC-DFis-UFPR.
[1] A. Y. Loskutov, J. Phys. A: Math. Gen. 26, 4581 (1993).

[2] K. A. Mirus and J. C. Sprott, Phys. Rev. E 59, 5313 (1999).

[3] N. Inaba, M. Sekikawa, T. Endo, and T. Tsubouchi, Int. J. Bif. Chaos 13, 2905 (2003).

[4] E. Ott, Chaos in Dynamical Systems (Cambridge, 2002).

[5] Z. Qu, G. Hu, G. Yang, and G. Qin, Phys. Rev. Lett. 74, 1736 (1995).

[6] A. Y. Loskutov, S. D. Rybalko, U. Feudel, and J. Kurths, J. Phys. A: Math. Gen. 29, 5759 (1996).

[7] Sanju and V. S. Varma, Phys. Rev. E 48, 1670 (1993).

[8] T. Buchner and J. J. Żebrowski, Phys. Rev. E 63, 016210 (2000).

[9] A. Nandi, D. Dutta, J. K. Bhattacharjee, and R. Ramaswamy, Chaos 15, 023107 (2005).

[10] D. R. da Costa, M. Hansen, G. Guarise, R. O. MedranoT, and E. D. Leonel, Phys. Lett. A 380, 1610 (2016).

[11] H. Aref, J. R. Blake, M. Budišić, S. S. S. Cardoso, J. H. E.
Cartwright, H. J. H. Clercx, K. El Omari, U. Feudel, R. Golestanian, E. Gouillart, et al., Rev. Mod. Phys. 89, 025007 (2017).

[12] R. M. Noyes, J. Chem. Phys. 64, 1266 (1976).

[13] J. A. C. Gallas, Physica A 222, 125 (1995).

[14] C. Manchein, A. Celestino, and M. W. Beims, Phys. Rev. Lett. 110, 114102 (2013).

[15] A. C. C. Horstmann, H. A. Albuquerque, and C. Manchein, Eur. Phys. J. B. 90, 96 (2017).

[16] C. Manchein, R. M. da Silva, and M. W. Beims, Chaos 27, 081101 (2017).

[17] A. Celestino, C. Manchein, H. A. Albuquerque, and M. W. Beims, Phys. Rev. Lett. 106, 234101 (2011).

[18] A. Celestino, C. Manchein, H. A. Albuquerque, and M. W. Beims, Commun. Nonlinear Sci. Numer. Simul. 19, 139 (2013).

[19] M. W. Beims, M. Schlesinger, C. Manchein, A. Celestino, A. Pernice, and W. T. Strunz, Phys. Rev. E 91, 052908 (2015). 